\title{
Comparative Effectiveness Research in Localized Prostate Cancer: A 10-Year Follow-up Cohort Study
}

Olatz Garin, PhD, ${ }^{*}, \downarrow$ José Francisco Suárez, MD, ${ }^{\S}$ Ferran Guedea, PhD, $\|$ Àngels Pont, MSc, ${ }^{*, \dagger}$ Yolanda Pardo, PhD, ${ }^{*},+\mathscr{T}$ Alai Goñi, MD, ${ }^{\#}$ Alfonso Mariño, MD, ** Asunción Hervás, PhD, ${ }^{\dagger \dagger}$ Ismael Herruzo, PhD, Patricia Cabrera, PhD, ${ }^{\$ \S}$ Gemma Sancho, PhD, \|\| Javier Ponce de León, PhD, ${ }^{\mp \top}$ Víctor Macías, PhD, \# Cristina Gutierrez, PhD, Manel Castells, MD, and Montse Ferrer, PhD, ${ }^{*, t, \pi}$ Multicentric Spanish Group of Clinically Localized Prostate Cancer

\begin{abstract}
*Health Services Research Group, Hospital del Mar Medical Research Institute, Barcelona, Spain; ${ }^{\dagger}$ CIBER en Epidemiologia y Salud Pública, CIBERESP,Spain; ${ }^{\ddagger}$ Pompeu Fabra University, Barcelona, Spain; ${ }^{\S}$ Hospital Universitari de Bellvitge, L'Hospitalet de Llobregat, Spain; "Institut Català d'Oncologia, L'Hospitalet de Llobregat, Spain; "Universitat Autònoma de Barcelona, Bellaterra, Spain; "Institutu Onkologikoa, San Sebastián, Spain; * *Servicio de Oncología Radioterápica, Centro Oncológico de Galicia, A Coruña, Spain; ${ }^{\dagger \dagger}$ Servicio de Oncología Radioterápica, Hospital Ramón y Cajal, Madrid, Spain; ${ }^{\ddagger}$ Servicio de Oncología Radioterápica, Hospital Regional Carlos Haya, Málaga, Spain; ${ }^{\S}$ Servicio de Oncología Radioterápica, Hospital Virgen del Rocio, Sevilla, Spain; ${ }^{\|\|}$Servicio de Oncología Radioterápica, Hospital Santa Creu i Sant Pau, Barcelona, Spain; " ${ }^{\top \uparrow}$ Servicio de Urología, Fundació Puigvert, Barcelona, Spain; and ${ }^{\# \#}$ Hospital Universitario y Politecnico La Fe, Valencia, Spain
\end{abstract}

Received Aug 12, 2020. Accepted for publication Dec 22, 2020.

Purpose: Long-term comparative effectiveness research on localized prostate cancer treatments is scarce, and evidence is lacking especially for brachytherapy. The aim of this study was to assess the long-term impact of the side effects of radical prostatectomy, brachytherapy, and external radiation therapy on patients with localized prostate cancer at 10 years, using propensity score analyses.

Corresponding author: Montse Ferrer, PhD; E-mail: mferrer@imim.es

This study was supported by grants from the Instituto de Salud Carlos III FEDER: Fondo Europeo de Desarrollo Regional (grant numbers PI02/ 0668, PI08/90090, and PI13/00412); Generalitat de Catalunya, Spain (grant numbers 086/24/2000 and 2017 SGR 452); and Ministerio de Ciencia e Innovación, Spain (grant number PTA2011-04891). The funders of the study had no role in the study design and conduct, data collection, management, analysis, or interpretation; they also had no role in the preparation, writing, reviewing, and submission of this manuscript.

Disclosures: The authors declare no potential conflicts of interest.

Study identifier at ClinicalTrials.gov is NCT01492751.
Data sharing statement: Data are available for bona fide researchers who request it from the authors.

Supplementary material for this article can be found at https://doi.org/ 10.1016/j.ijrobp.2020.12.032.

Acknowledgments - The authors acknowledge the collaboration and contribution of other members of the Multicentric Spanish Group of Clinically Localized Prostate Cancer. The authors also thank the patients and families who made this study possible and the clinical study teams who participated in the study. Moreover, the group thanks Aurea Martin for her support in English editing, proofreading, and preparing this manuscript for submission. 
Methods and Materials: This was a prospective observational study of a cohort of men who received a diagnosis of clinically localized prostate cancer (clinical stage T1 or T2, low and intermediate risk group) and were treated with radical prostatectomy $(\mathrm{n}=139)$, brachytherapy $(\mathrm{n}=317)$, or external radiation therapy $(\mathrm{n}=194)$. Treatment decisions were jointly made by patients and physicians. Patient-reported outcome (PRO) evaluation included the Expanded Prostate Cancer Index Composite and Short Form-36, administered centrally by telephone interviews before and annually after treatment. The Expanded Prostate Cancer Index Composite covers urinary, bowel, sexual, and hormonal domains. To assess PRO changes over time, while accounting for correlation among repeated measures, generalized estimating equation models adjusted by propensity scores were constructed.

Results: The PRO completion rate at 10 years was $85.8 \%$. Generalized estimating equation models showed that the pattern of radical prostatectomy side effects, with substantial urinary incontinence and sexual dysfunction, remained until 10 years after treatment (standard deviation $[\mathrm{SD}],-1.1$ and -1.3 , respectively). Brachytherapy produced late deterioration in urinary continence $(\mathrm{SD},-0.4)$ and sexual function $(\mathrm{SD},-0.9)$ that appeared midterm, but the differences from radical prostatectomy remained statistically significant at 10 years $(P<.001$ after adjusting by propensity score). External radiation therapy showed similar results to brachytherapy, but with bowel bother $(\mathrm{SD},-0.3)$.

Conclusions: Although late deterioration in radiation therapy groups attenuated differences from radical prostatectomy, relevant PRO differences still remained after 10 years. Our findings support that brachytherapy is the treatment option that causes the least impact on PROs; it is therefore an alternative to be considered when making evidence-based decisions on localized prostate cancer treatment. (C) 2021 The Authors. Published by Elsevier Inc. This is an open access article under the CC BYNC-ND license (http://creativecommons.org/licenses/by-nc-nd/4.0/).

\section{Introduction}

Prostate cancer is the most frequently diagnosed cancer among men in the United States and Europe. ${ }^{1}$ Currently, most patients with prostate cancer are diagnosed in localized stages ${ }^{2}$ and will probably be long-term survivors. ${ }^{3}$

Randomized controlled trials of curative intention treatments for localized prostate cancer are mainly restricted to the Prostate Testing for Cancer and Treatment (ProtecT) trial. ${ }^{4,5}$ This study showed similar very high rates of survival at 10 years of follow-up ${ }^{4}$ for radical prostatectomy, external radiation therapy, and active monitoring; however, treatments differed in side-effect patterns as measured by patient-reported outcomes (PROs). ${ }^{5}$ These results have been confirmed by an analysis of the trial results according to the treatment received and including patients who refused the randomization. ${ }^{6}$ Long-term PRO follow-ups have been also reported by the Cancer of the Prostate Strategic Urologic Research Endeavor (CaPSURE) ${ }^{7}$ trial and Prostate Cancer Outcomes Study, ${ }^{8}$ but CaPSURE is the only study to evaluate brachytherapy.

A systematic review of PROs in clinically localized prostate cancer treatments ${ }^{9}$ identified 6 studies evaluating brachytherapy with follow-ups of 5 to 6 years, and their combined results indicated a reduced adverse effect profile. In contrast, 5 and 10 years after treatment, patients undergoing brachytherapy in the CaPSURE study ${ }^{7}$ presented a similar risk of sexual deterioration compared with those who received radical prostatectomy, a lower risk of urinary function worsening, and a higher risk of bowel dysfunction. Since that review and up to the present, no other study on brachytherapy has shown relevant evidence on this treatment. Three articles have published data at 12 to $14,{ }^{10} 8,{ }^{11}$ and 8 to $9^{12}$ years of follow-up, but they presented relevant limitations: a small sample size $\left(42^{10}\right.$ or $6^{12}$ patients in the brachytherapy group at the last follow-up), a very low response rate $(13 \%),{ }^{11}$ or only covering bowel impact. ${ }^{12}$ Therefore, evidence on brachytherapy remains scarce and controversial in the long term.

The aim of this study was to assess the long-term impact of the side effects of radical prostatectomy, brachytherapy, and external radiation therapy on patients with localized prostate cancer 10 years after treatment, using propensity score analyses.

\section{Methods and Materials}

This was a prospective observational study (ClinicalTrials. gov Identifier: NCT01492751) of a cohort of men who received a diagnosis of clinically localized prostate cancer and were treated with radical prostatectomy $(\mathrm{n}=193)$, brachytherapy ( $\mathrm{n}=317)$, or external radiation therapy ( $\mathrm{n}$ $=194$ ). Study details have been described elsewhere. ${ }^{13-15}$ Briefly, eligible patients were those with clinical stage T1 or T2, low and intermediate D' Amico risk group disease, ${ }^{16}$ treated at one of the participating centers, and without previous prostate transurethral resection. Decisions regarding treatment were made jointly by patients and physicians. The study was approved by the ethics review boards of the participating hospitals, and written informed consent was obtained from patients per the 2000 revision of the Helsinki Declaration.

The Expanded Prostate Cancer Index Composite (EPIC) ${ }^{17,18}$ and Short Form-36 (SF-36) ${ }^{19,20}$ were administered centrally by telephone interviews before treatment and during follow-up at $1,3,6$, and 12 months after treatment during the first year and annually thereafter. EPIC covers urinary, bowel, sexual, and hormonal domains, with 
50 items and scores ranging from 0 to $100 .{ }^{17}$ The SF-36 (version 2) generates physical and mental component summaries, scored as recommended by developers and standardized to have a mean of 50 and standard deviation (SD) of 10 in the general US population. ${ }^{19}$ Higher scores indicate better results in both instruments. In addition to the EPIC scores, we selected the key EPIC items already identified in the ProtecT study ${ }^{5}$ to aid in the interpretation of clinical relevance. Responses to these key items were all dichotomized to show the percentage of men reporting problems, except for the erection firmness item (percentage of men reporting sexual potency).

The sample size was calculated to detect between-group differences of 5 points on the urinary irritative-obstructive score of the EPIC questionnaire. ${ }^{13}$

\section{Statistical analysis}

Summary statistics and 95\% confidence intervals (CIs) are reported by treatment group. To account for treatment selection bias, previously estimated propensity scores, ${ }^{15}$ which had already been demonstrated to balance the distribution of possible confounders among treatment groups, were incorporated into multivariate analyses.

To assess PRO changes over time, while accounting for correlation among repeated measures, separate generalized estimating equation (GEE) models were constructed for each EPIC score or key item (included as dependent variables). Time was included in the model as a categorical variable, and interactions between treatment and time were considered to test differences on trends among the treatment groups. The GEE models were constructed with the use of SAS/STAT software, version 9.4.

\section{Results}

At baseline, the treatment groups presented statistically significant differences in age (means from 64.2 years in radical prostatectomy to 70.1 years in external radiation therapy; $P<.001$ ), prostate-specific antigen level (means from $7.0 \mathrm{ng} / \mathrm{mL}$ in brachytherapy to $8.1 \mathrm{ng} / \mathrm{mL}$ in external radiation therapy; $P<.001$ ), and Gleason score (means from 5.5 in brachytherapy to 6.3 in radical prostatectomy; $P<.001)$. After propensity score adjustment, ${ }^{15}$ the differences in baseline clinical characteristics among treatment groups disappeared.

The flow chart in Figure E1 shows that at 10 years after treatment, 149 of 704 participants had died, 74 missed the interview, 5 were lost to follow-up, and 476 completed the PRO questionnaires. The completion rate at 10 years was 85.8\% (median, 10.24 years; interquartile range, 10.28-10.14).

Figure 1 shows the annual results of the EPIC urinary domain with unadjusted means or percentages with their 95\% CI and $P$-values adjusted by propensity scores. Urinary incontinence (Fig. 1A) presented statistically significant time-trend differences among the treatment groups, with patients who underwent radical prostatectomy presenting the greatest deterioration. The use of absorbent pads (Fig. 1B) was null before treatment and increased 10 years after to $34.3 \%$ in the prostatectomy group, $6.9 \%$ in the brachytherapy group, and $7.8 \%$ in the external radiation therapy group. Although the urinary irritative-obstructive score was quite stable during follow-up (Fig. 1C), the radiation therapy groups presented statistically lower (worse) means than radical prostatectomy. Figure 1D shows the rate of men reporting pain or burning with urination, which was quite low during the whole follow-up ( $<7 \%$ for all groups).

All scores and selected key items from the EPIC sexual domain showed statistically significant different results over 10 years of follow-up across the treatment groups (Fig. 2). The sexual function score decreased (deteriorated) with radical prostatectomy and external radiation therapy, but patients treated with brachytherapy showed stability during the first 5 years after treatment and deterioration thereafter (Fig. 2A). Before surgery, 58.1\% of men reported erections firm enough for intercourse, but this rate fell to 9.4\% after 1 year (Fig. 2B). In the brachytherapy group, this rate remained stable at around $40 \%$ from before treatment to the fifth year and declined thereafter.

Figure 3 shows that only the key item about loose stools (Fig. 3B) and the bowel summary score (Fig. 3G) presented statistically significant differences among treatments over time $(P=.027$ and $P=.032)$, both indicating worse results in the external radiation therapy group. Calculating the $P$-value of trend differences for fecal incontinence (Fig. 3C) and bloody stools (Fig. 3D) was not possible, because in some evaluations no men reported these problems.

Figure 4 shows the changes at 10 years obtained by GEE models constructed to assess treatment impact on PROs after adjusting by propensity scores. The GEE models with all the coefficients and $P$-values are available in the Table E1. Units of change on the left of the figure are EPIC scores and those on the right SDs (change: moderate $=0.5$ $\mathrm{SD}$ and large $=1 \mathrm{SD}$, indicated by gray lines). ${ }^{21} \mathrm{On}$ average, 10 years after radical prostatectomy, patients scored 29.9 points less than before treatment (Fig. 4A). This change of 30 points is greater than 1 SD (large deterioration). Patients treated with brachytherapy fared +17.4 points (95\% CI, 11.0-23.9) better (positive sign) than those who underwent radical prostatectomy (reference group) and, similarly, those who underwent external radiation therapy. Urinary continence deterioration in the brachytherapy and external radiation therapy groups has an near $0.5 \mathrm{SD}$ (moderate deterioration).

Figure $4 \mathrm{~B}$ shows that both radiation therapy groups presented worse results than radical prostatectomy on the urinary irritative-obstructive score $(-3.9$ points for brachytherapy; -5.5 points for external radiation therapy). Figure $4 \mathrm{C}$ shows substantial worsening of the EPIC sexual function score at 10 years in the 3 treatment groups, but patients treated with brachytherapy fared +10.8 points (95\% CI, 4.8-16.8) better than those in the radical 


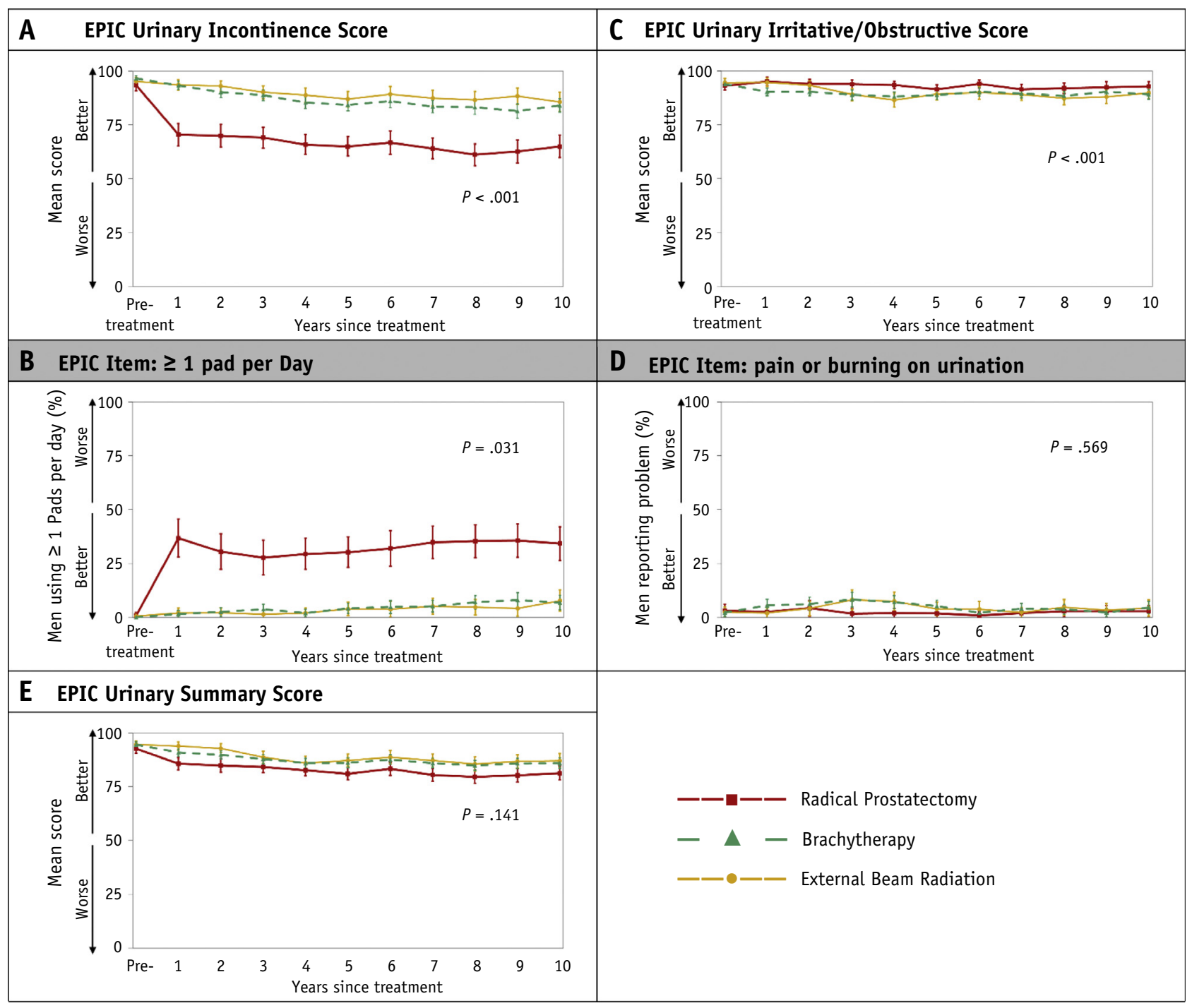

Fig. 1. Ten years of annual results in the urinary domain. Effects of treatments on the urinary domain measured with the Expanded Prostate Cancer Index Composite (EPIC) instrument. EPIC urinary scores comprise several urinary items and are formed by linear transformation of raw scores, ranging from 0 to 100 (from worse to better). (A) EPIC score for urinary incontinence. (B) Results for one of the items included in that score: Percentage of men who used $\geq 1$ absorbent pads per day for urinary incontinence. (C) EPIC urinary irritative-obstructive score. (D) Percentages for men who reported moderate-tosevere pain or burning on urination. (E) EPIC urinary summary score. $P$-values show the strength of evidence for a difference in mean response over 10 years of follow-up across the 3 groups, adjusted by propensity score. I bars represent unadjusted $95 \%$ confidence intervals.

prostatectomy group. Finally, worsening was not observed in the bowel function domain (Fig. 4E), and the small worsening presented in bowel bother $(<0.5 \mathrm{SD})$ did not statistically differ among treatments (Fig. 4F).

\section{Discussion}

Our findings show that the main traits of radicalprostatectomy side-effect patterns, with large urinary incontinence and sexual dysfunction, remain until 10 years after treatment. Brachytherapy produced substantial deterioration in sexual function and moderate urinary incontinence that appears at midterm (5 and 7 years after treatment). External radiation therapy showed results similar to brachytherapy, but with bowel bother. Although late deterioration in the radiation therapy groups attenuated their differences from radical prostatectomy, they remained relevant at 10 years.

The main limitation of this study is its observational design. The main concern regarding this design is treatment selection bias where, for example, brachytherapy is preferentially prescribed to patients with lower tumor risk and surgery to younger patients. ${ }^{13-15}$ In our cohort, the propensity scores balanced treatment selection bias. ${ }^{15}$ Results 


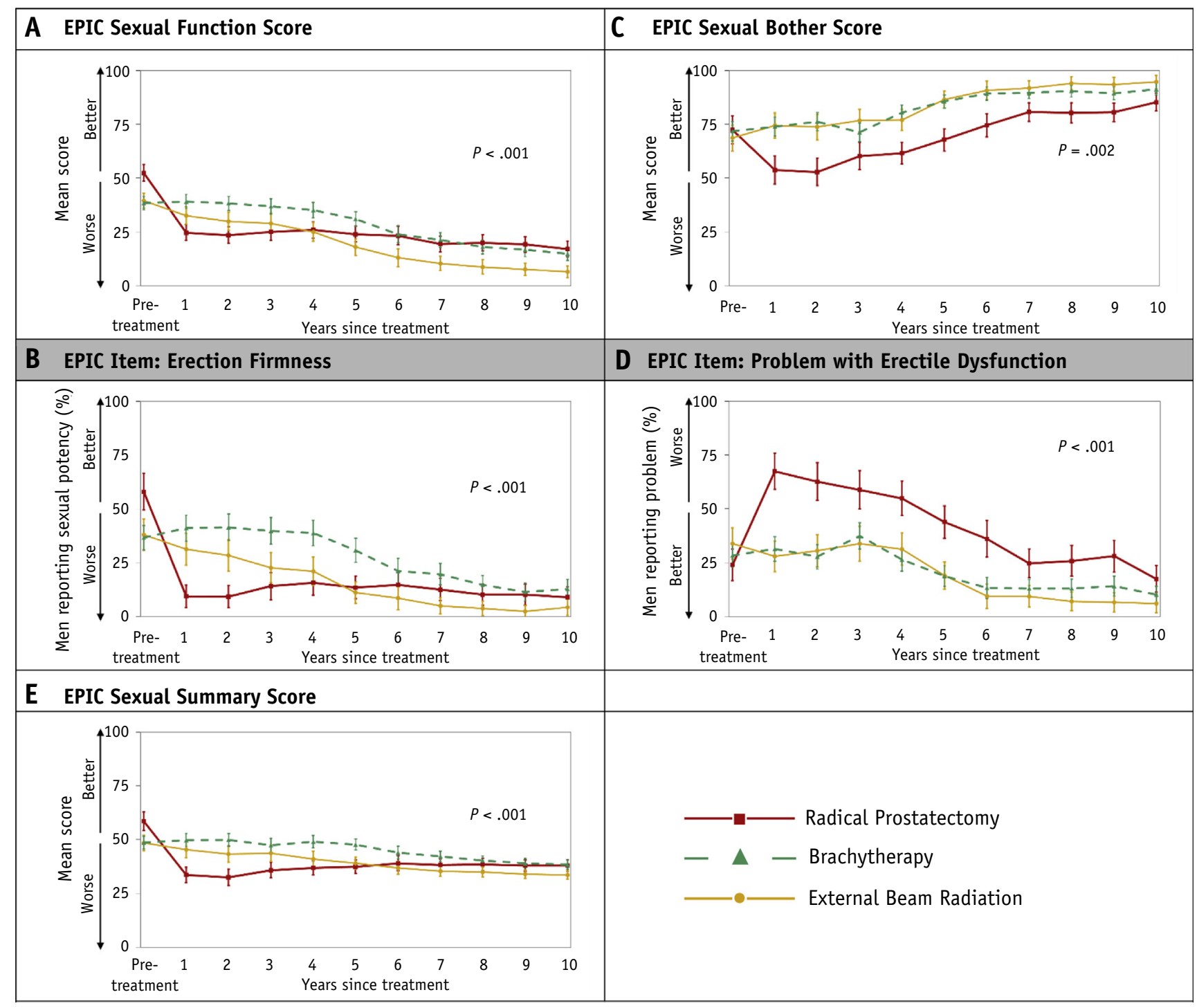

Fig. 2. Ten years of annual results in the sexual domain. Effects of treatments on the sexual domain measured with the Expanded Prostate Cancer Index Composite (EPIC) instrument. EPIC sexual scores comprise several sexual items and are formed by linear transformation of raw scores, ranging from 0 to 100 (from worse to better). (A) EPIC sexual function score. (B) Results for one of the items included in this score: Percentage of men reporting erections firm enough for intercourse. (C) EPIC sexual bother score. (D) Percentages for men who reported a moderate-to-severe problem with erectile dysfunction. (E) EPIC sexual summary score. $P$-values show the strength of evidence for a difference in mean response over 10 years of follow-up across the 3 groups, adjusted by propensity score. I bars represent unadjusted $95 \%$ confidence intervals.

adjusted by propensity scores were consistent with those from traditional models adjusted by baseline clinical variables (Table E2), likely allowing an estimation of the true treatment effects. ${ }^{22}$ Second, the treatments were applied $>1$ decade ago; since then, diagnostic techniques and treatments for prostate cancer have evolved.

The strengths of the study include the collection of data per the present recommendations of the International Consortium for Health Outcomes Measurement for localized prostate cancer, which were developed in $2015,{ }^{23}$ the use of a propensity score adjustment for comparative effectiveness research, ${ }^{22}$ regular follow-up with high rates of response (median, 83.3\%; interquartile range, 85.7-74.0), and the consistency of interpretation based on the magnitude of change with previously established minimally important differences for EPIC scores. ${ }^{24}$ This is the first study to provide robust evidence on the long-term outcomes of brachytherapy because only 85 of 684 patients treated with this therapy in the CaPSURE registry were evaluated 10 years after treatment. ${ }^{7}$ Of the 317 patients treated with brachytherapy in our cohort, 69 died and 217 completed the 10 -year evaluation.

CaPSURE applied the University of California, Los Angeles, Prostate Cancer Index to measure clinically meaningful declines in PROs (defined as 0.5 SD) after treatment. ${ }^{7}$ To facilitate the comparison with CaPSURE, we 


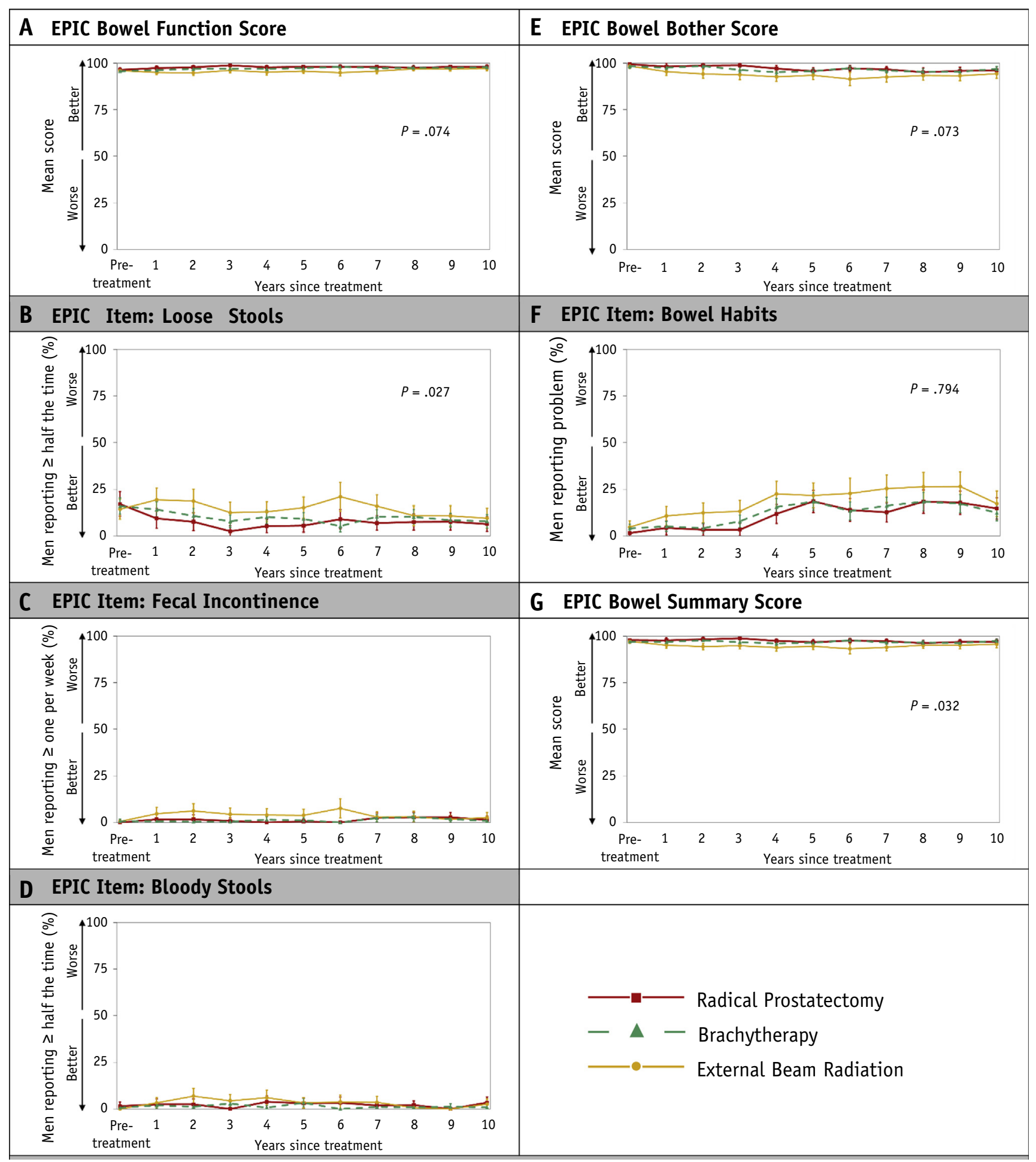

Fig. 3. Ten years of annual results in the bowel domain. Effects of the treatments on the bowel domain measured with the Expanded Prostate Cancer Index Composite (EPIC) instrument. EPIC bowel scores comprise several bowel items and are formed by linear transformation of raw scores, ranging from 0 to 100 (from worse to better). (A) EPIC score for bowel function. (B-D) Results for items included in that score as percentages of men who reported having (B) loose stools half the time or more, (C) fecal incontinence at least once per week, and (D) bloody stools half the time or more. (E) EPIC bowel bother score. (F) Percentages for men who reported a moderate-to-severe negative effect on bowel habits. (G) EPIC bowel summary score. $P$-values show the strength of evidence for a difference in mean response over 10 years of follow-up across the 3 groups, adjusted by propensity score. I bars represent unadjusted 95\% confidence intervals. 
Radical Prostatectomy

A EPIC Urinary Incontinence Score

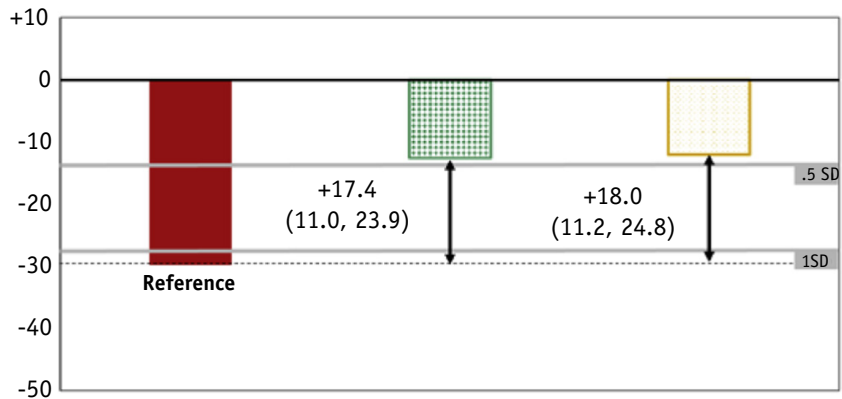

C EPIC Sexual Function Score

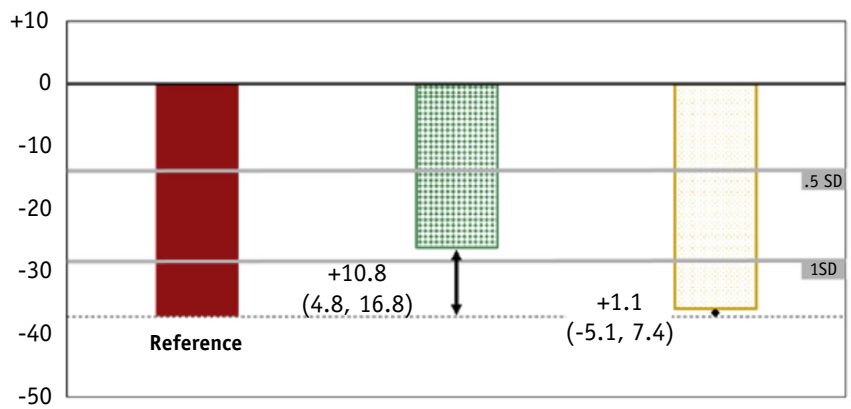

E EPIC Bowel Function Score

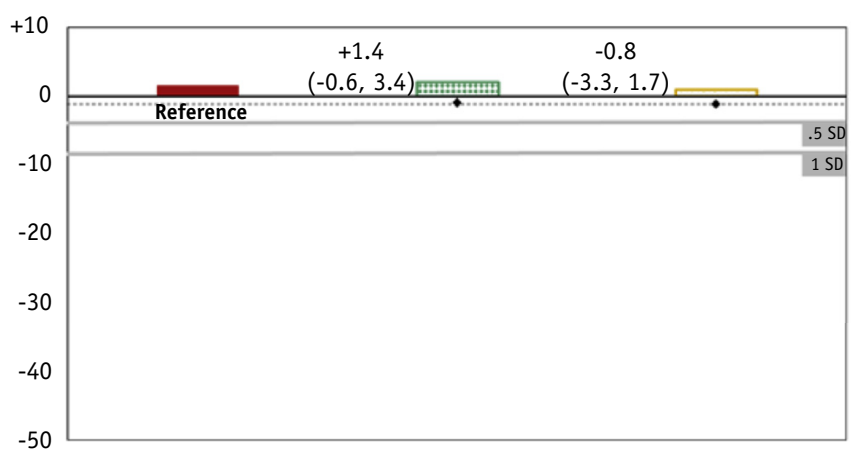

External Radiotherapy

\section{B EPIC Urinary Irritative/Obstructive Score}

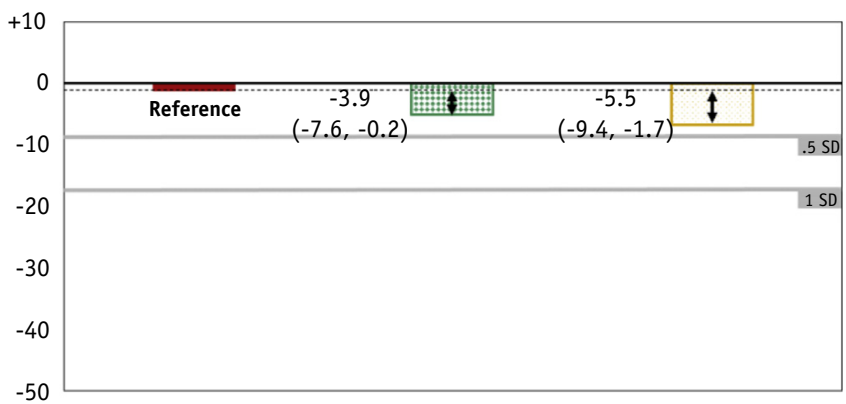

D EPIC Sexual Bother Score

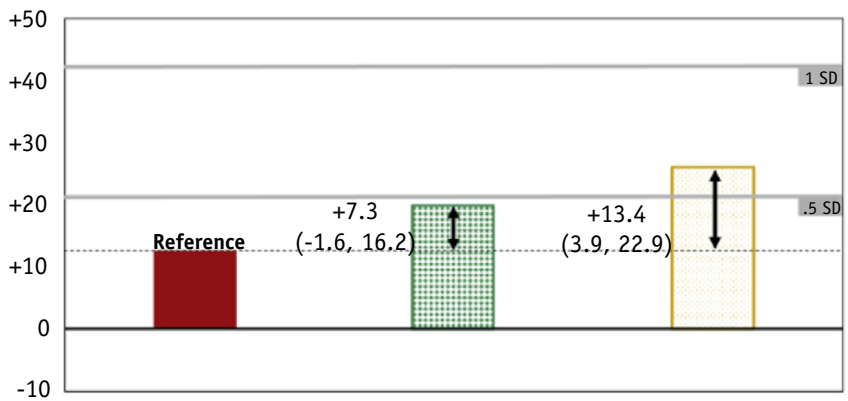

F EPIC Bowel Bother Score

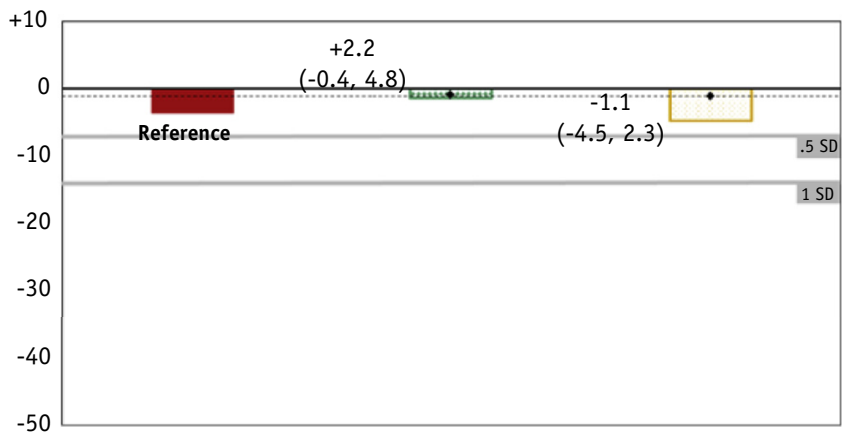

Fig. 4. Impact on patient-reported outcomes 10 years after treatment. On the left side of the figures, the units of change are EPIC scores and on the right side, they are standard deviations (moderate $=0.5 \mathrm{SD}$; large $=1 \mathrm{SD}$, indicated by gray lines). Mean changes in Expanded Prostate Cancer Index Composite instrument scores from pretreatment to 10 years of follow-up, obtained by generalized estimating equation models, are adjusted by propensity scores. Each bar is complemented with the difference compared with radical prostatectomy (mean and 95\% confidence interval).

similarly calculated odds ratios adjusted by propensity scores (Table E3) with radical prostatectomy as the reference group. Because EPIC was derived from the University of California, Los Angeles, Prostate Cancer Index, mainly by transforming the urinary domain, only the sexual and bowel domains are comparable. The results for sexual function were similar for the radiation therapy groups, and differences were not statistically significant at 10 years in our cohort, nor in the CaPSURE study, with an odds ratio of 0.8 (95\% CI, 0.3-1.7) and $0.9(95 \% \mathrm{CI}, 0.3-2.6)^{7}$ in brachytherapy and $1.3(95 \% \mathrm{CI}$, $0.5-3.2)$ and 0.7 (95\% CI, 0.2-3.4) $)^{7}$ in external radiation therapy. Our bowel results in the external radiation therapy group were also similar to those reported by CaPSURE, but not in the brachytherapy group.

The probability of experiencing a meaningful decline after brachytherapy compared with radical prostatectomy at 10 years after treatment was higher among the CaPSURE patients than in our cohort, with odds ratios of 3.3 (95\% CI, $1.4-7.4)^{7}$ versus 2.3 (95\% CI, 0.8-6.7) in bowel function and 2.3 (95\% CI, 1.0-5.2) ${ }^{7}$ versus 1.5 (95\% CI, 0.5-4.0) in bowel bother. Therefore, discrepancies compared with CaPSURE were mainly restricted to the better results obtained in our study for brachytherapy in the bowel domain. Although CaPSURE is a nationwide prostate cancer registry, it is 
important to highlight the low number of patients in the brachytherapy group with results at 10 years ${ }^{7}$ ranged from 76 to 85 according to the domain evaluated. Follow-up losses could have produced some bias in CaPSURE, which may explain the differences when compared with our study. For example, completion rate could be higher among patients with worse PRO results, because patients without problems may tend to disregard clinical encounters.

In addition, patients who underwent brachytherapy in our cohort presented a similar sexual pattern to results reported by Keyes et $\mathrm{al}^{25}$ in a large cohort of almost 3000 patients followed for a median of 3.5 years and a maximum of 14 years, when focusing on patients with full potency before treatment and without neoadjuvant androgen deprivation therapy: a gradual and continuous erectile function decline. In our study, the percentage of men reporting sexual potency in this selected subsample went from $64 \%$ at 1 year after treatment to $53 \%$ after 4 years and $34 \%$ after 7 years. In the study by Keyes et al, the most significant drop in erectile function among patients with full potency before treatment was also during the first months: approximately $65 \%$ reported full potency 1 year after treatment, approximately $55 \%$ at 4 years, and $40 \%$ at 7 years after treatment. ${ }^{25}$ This similarity is especially noteworthy because the erectile function measurement in both studies has a different nature. Ours is an item response in a PRO (EPIC), and Keyes et al assessed erectile function through a physician-reported 3-point scale. ${ }^{25}$

Our long-term findings in PROs confirm those reported by the ProtecT trial at 6 years ${ }^{5,6}$ regarding the distinct impact of localized prostate-cancer treatment side effects, but similar general health. Figure E2 and Table E1 show the stability of the SF-36 summaries until the sixth year of follow-up. Both studies show a very similar negative effect of prostatectomy on sexual domain (ie, only $14.7 \%$ of men reported sexual potency at year 6 in our study and $15 \%$ in the ProtecT trial). ${ }^{6}$ Regarding radical prostatectomy's impact on urinary continence, the percentage of patients who required the use of pads was lower in the ProtecT trial than in our cohort ( $21 \%^{6}$ vs $32 \%$ by year 6$)$. In both studies, external radiation therapy is related to bowel bother and function deterioration at 6 years after treatment (ie, $5.9 \%$ of men reporting bloody stools more than half the time in the ProtecT trial ${ }^{6}$ and $3.8 \%$ in our study).

Of note, the ProtecT trial's inclusion criteria were limited to patients diagnosed by screening. As a consequence, the baseline tumor characteristics of patients in the ProtecT trial and our cohort are very different, including median prostate-specific antigen levels of 4.8 versus 8.1 $\mathrm{ng} / \mathrm{mL}, 78 \%$ versus $43.3 \%$ of patients with Gleason score 6 , and $79 \%$ versus $58.2 \%$ with clinical T1 stage. ${ }^{4}$

\section{Conclusions}

Novel long-term results are provided on the impact of brachytherapy adverse effects in patients with localized prostate cancer 10 years after treatment compared with radical prostatectomy and external radiation therapy. Our findings support that, despite some late deterioration in urinary continence and sexual function around 5 years after treatment, brachytherapy is the treatment option causing the least impact on PROs. These results provide patients, clinicians, and health care planners with clear information to make evidence-based decisions and facilitate shared clinical decision-making, taking into account patients' perspective. Although no single treatment can be considered the preferred strategy for managing all patients, our study supports brachytherapy as a possible alternative for patients with localized prostate cancer seeking attempted curative treatment while avoiding bowel effects and postponing the risk for urinary incontinence and sexual dysfunction.

\section{References}

1. Bray F, Ferlay J, Soerjomataram I, Siegel RL, Torre LA, Jemal A. Global cancer statistics 2018: GLOBOCAN estimates of incidence and mortality worldwide for 36 cancers in 185 countries. CA Cancer J Clin 2018;68:394-424.

2. Shao YH, Demissie K, Shih W, et al. Contemporary risk profile of prostate cancer in the United States. J Natl Cancer Inst 2009;101: 1280-1283.

3. Chou R, Croswell JM, Dana T, et al. Screening for prostate cancer: A review of the evidence for the U.S. Preventive Services Task Force. Ann Intern Med 2011;155:762-771.

4. Hamdy FC, Donovan JL, Lane JA, et al. 10-year outcomes after monitoring, surgery, or radiotherapy for localized prostate cancer. $N$ Engl J Med 2016;375:1415-1424.

5. Donovan JL, Hamdy FC, Lane JA, et al. Patient-reported outcomes after monitoring, surgery, or radiotherapy for prostate cancer. $N$ Engl J Med 2016;375:1425-1437.

6. Neal DE, Metcalfe C, Donovan JL, et al. Ten-year mortality, disease progression, and treatment-related side effects in men with localised prostate cancer from the ProtecT randomised controlled trial according to treatment received. Eur Urol 2020;77:320-330.

7. Punnen S, Cowan JE, Chan JM, Carroll PR, Cooperberg MR. Longterm health-related quality of life after primary treatment for localized prostate cancer: Results from the CaPSURE registry. Eur Urol 2015; 68:600-608.

8. Resnick MJ, Koyama T, Fan KH, et al. Long-term functional outcomes after treatment for localized prostate cancer. $N$ Engl J Med 2013;368:436-445.

9. Ávila M, Patel L, López S, et al. Patient-reported outcomes after treatment for clinically localized prostate cancer: A systematic review and meta-analysis. Cancer Treat Rev 2018;66:23-44.

10. Jang JW, Drumm MR, Efstathiou JA, et al. Long-term quality of life after definitive treatment for prostate cancer: Patient-reported outcomes in the second posttreatment decade. Cancer Med 2017;6:18271836.

11. Ling DC, Chen KS, Benoit RM, et al. Long-term patient-reported rectal bleeding and bowel-related quality of life after Cs-131 prostate brachytherapy. Int J Radiat Oncol Biol Phys 2019;104:622-630.

12. Frank SJ, Pugh TJ, Blanchard P, et al. Prospective phase 2 trial of permanent seed implantation prostate brachytherapy for intermediaterisk localized prostate cancer: Efficacy, toxicity, and quality of life outcomes. Int J Radiat Oncol Biol Phys 2018;100:374-382.

13. Ferrer M, Suarez JF, Guedea F, et al. Health-related quality of life 2 years after treatment with radical prostatectomy, prostate 
brachytherapy, or external beam radiotherapy in patients with clinically localized prostate cancer. Int J Radiat Oncol Biol Phys 2008;72: 421-432.

14. Pardo Y, Guedea F, Aguilo F, et al. Quality-of-life impact of primary treatments for localized prostate cancer in patients without hormonal treatment. J Clin Oncol 2010;28:4687-4696.

15. Ferrer M, Guedea F, Suarez JF, et al. Quality of life impact of treatments for localized prostate cancer: cohort study with a 5 year followup. Radiother Oncol 2013;108:306-313.

16. D'Amico AV, Whittington R, Malkowicz SB, et al. Biochemical outcome after radical prostatectomy, external beam radiation therapy, or interstitial radiation therapy for clinically localized prostate cancer. JAMA 1998;280:969-974.

17. Wei JT, Dunn RL, Litwin MS, Sandler HM, Sanda MG. Development and validation of the expanded prostate cancer index composite (EPIC) for comprehensive assessment of health-related quality of life in men with prostate cancer. Urology 2000;56: 899-905.

18. Ferrer M, Garin O, Pera J, et al. Evaluation of the quality of life of patients with localized prostate cancer: Validation of the Spanish version of the EPIC. Med Clin (Barc) 2009;132:128-135.
19. Ware JE, Kosinski M, Dewey JE. How to Score. Version 2 of the SF-36 Health Survey (Standard \& Acute Forms). Lincoln RI: Quality Metric Incorporated; 2000;1-231.

20. Alonso J, Prieto L, Anto JM. La versión española del "SF-36 Health Survey" (Cuestionario de Salud SF-36): Un instrumento para la medida de los resultados clínicos. Med Clin (Barc) 1995;104:771-776.

21. Cohen J. Statistical power analysis for the behavioral sciences. 2nd Edition. Hillsdale, NJ: Lawrence Elrbaum Associates; 1988;1-559.

22. Desai RJ, Franklin JM. Alternative approaches for confounding adjustment in observational studies using weighting based on the propensity score: A primer for practitioners. BMJ 2019;367:15657.

23. Martin NE, Massey L, Stowell C, et al. Defining a standard set of patient-centered outcomes for men with localized prostate cancer. Eur Urol 2015;67:460-467.

24. Umbehr MH, Bachmann LM, Poyet C, et al. The German version of the Expanded Prostate Cancer Index Composite (EPIC): Translation, validation and minimal important difference estimation. Health Qual Life Outcomes 2018;16:36.

25. Keyes M, Pickles T, Crook J, et al. Effect of aging and long-term erectile function after iodine-125 prostate brachytherapy. Brachytherapy 2015;14:334-341. 